\title{
Spectra of $O$ II in the plasma boundary of TEXTOR-94
}

\author{
D Borodin ${ }^{1,2}$, I L Beigman ${ }^{2}$, L A Vainshtein ${ }^{2}$, A Pospieszczyk ${ }^{1}$, \\ S Brezinsek ${ }^{1}$ and A Huber ${ }^{1}$ \\ ${ }^{1}$ Institut fuer Plasmaphysik, Forschungszenrum Juelich, 52425 Juelich, Germany \\ 2 PN Lebedev Physical Institute RAS, Leninski pr. 53, 117924 Moscow, Russia \\ E-mail: d.borodin@fz-juelich.de
}

Received 18 December 2001, in final form 21 June 2002

Published 27 September 2002

Online at stacks.iop.org/PPCF/44/2251

\begin{abstract}
Spectra of the $\mathrm{O}_{\text {II }}$ ion were obtained with temporal and spatial (along the poloidal radius) resolution by injecting of oxygen-containing molecules through a block of the upper poloidal limiter of TEXTOR-94 into the boundary plasma. Three different wavelength regions $(4660 \pm 100,4340 \pm 100,3730 \pm 100 \AA)$ were observed during reproducible TEXTOR-94 discharges with $n_{\mathrm{e}}=1 \times 10^{18} \mathrm{~m}^{-3}$, $T_{\mathrm{e}}=90 \mathrm{eV}$ at the last closed flux surface (LCFS). For calibration purposes the oxygen emission was simultaneously registered in all discharges with a $2 \mathrm{D}$ camera using the light from an $\mathrm{O}_{\text {II }}$ line at $4416 \AA$. Radial intensity distributions of lines corresponding to the transitions $2 p^{2}\left[{ }^{3} \mathrm{P}\right] 3 \mathrm{~s}-2 \mathrm{p}^{2}\left[{ }^{3} \mathrm{P}\right] 3 \mathrm{p}$ (components of the multiplets ${ }^{4} \mathrm{P}-{ }^{4} \mathrm{~S},{ }^{4} \mathrm{P}-{ }^{4} \mathrm{P},{ }^{4} \mathrm{P}-{ }^{4} \mathrm{D},{ }^{2} \mathrm{P}-{ }^{2} \mathrm{D}$ ) and $2 \mathrm{p}^{2}\left[{ }^{1} \mathrm{D}\right] 3 \mathrm{~s}-2 \mathrm{p}^{2}\left[{ }^{1} \mathrm{D}\right] 3 \mathrm{p}$ (doublet $\left.{ }^{2} \mathrm{D}-{ }^{2} \mathrm{D}\right)$ were measured. It is shown that the relative intensities of the lines inside the multiplets are in good agreement with theoretical data calculated in SL-coupling. An appropriate collisional-radiative model (the level list and the corresponding atomic database) has been developed and calculations have been carried out using the GKU kinetic code developed at the P.N. Lebedev Institute. Cross-sections of atomic processes have been calculated using the ATOM code by the K-matrix method. A comparison of theoretical and experimental data is presented. It is shown that the relative multiplet intensities have a weak dependence on electron density and temperature. The populations of the ground configuration states of $\mathrm{O}$ II were investigated. Absolute values for the 'ionization per photon' were measured and a comparison with modelled values is given.
\end{abstract}

\section{Introduction}

Investigation of processes in the boundary plasma of devices for magnetic plasma confinement (tokamaks, stellarators) is very important, because such processes can affect the overall behaviour of the plasma. Important parameters for plasma confinement are the fluxes of different impurities from the wall into the plasma; oxygen is one such impurity. Spectral line 
intensity profiles can be used to estimate the oxygen flux [7]. Oxygen is released from the wall as atoms or even molecules and is ionized inside the plasma volume. The most important mechanisms of oxygen release inside the tokamak are [8]:

(a) Chemical release of $\mathrm{CO}$ and $\mathrm{CO}_{2}$ by bombardment of the surface of the tokamak by oxygen atoms (many parts of the modern tokamaks are made of graphite or at least covered by $\mathrm{C}$ containing coatings).

(b) Sputtering of other oxides from the surface and their release.

(c) Desorption (e.g. photon induced or ion bombardment induced) of oxygen-containing molecules $\left(\mathrm{H}_{2} \mathrm{O}, \mathrm{CO}, \mathrm{CO}_{2}\right)$ out the bulk material (graphite).

The strength of these processes depends on the boundary conditions: the concentration of water inside the vessel, the construction and chemical composition of the plasma facing components, radiation level, etc. Therefore, the situation can change from one discharge to another or even during one discharge.

The most direct way to determine the total flux of oxygen into the plasma is to use the diagnostics based on $\mathrm{O}_{\mathrm{I}}$ spectral lines. However, it was found that in TEXTOR all O I lines lying in the visible range are very weak or blended with $\mathrm{O}_{\text {II }}$ or other impurities lines. The strongest lines situated in the IR range (components of $2 s^{2} 2 p^{3}\left[{ }^{4} S\right] 3 s-2 s^{2} 2 p^{3}\left[{ }^{4} S\right] 3 p$ multiplet situated near $8500 \AA$ ) can be strongly influenced by continuum radiation, because limiters and even the liner can become hot. For the highly time resolved (below ms) measurements of fluxes usually diagnostics based on interference filters with transmission spectral ranges of about $30 \AA$ are used. The filter technique has a number of advantages for flux diagnostics, since it is stable, reliable and simple in use. However, with this kind of technique it is not possible to exclude the background produced by the 'black body'-like radiation of different tokamak parts changing strongly during one discharge. The type of oxygen release mechanism can, in principle, affect the $\mathrm{O}$ II spectra, because if, for instance, dissociation takes place, the initial population of the ion states can be different from the situation of ionization of neutrals. However, in this case there will also be a dependence on the type of the gas. This dependence should be examined.

Another possibility for the flux diagnostic is to use the $\mathrm{O}_{\text {II }}$ lines instead. Recently, the $2 p^{2}\left[{ }^{3} \mathrm{P}\right] 3 \mathrm{~s}^{4} \mathrm{P}-2 \mathrm{p}^{2}\left[{ }^{3} \mathrm{P}\right] 3 \mathrm{p}^{4} \mathrm{D}$ lines of $\mathrm{O}_{\text {II }}$ were studied with high spectral resolution in the tokamak TEXTOR [3]. In this work additional spectra of $\mathrm{O}_{\text {II }}$ in the blue visible wavelength range (which is less influenced by the black body radiation) were obtained in the boundary plasma of TEXTOR after the injection of oxygen-containing molecules near the last closed flux surface (LCFS). Several O II multiplets were identified and found to be suitable for evaluation. The estimation of the oxygen flux requires the construction of a collisional-radiative model (atomic database, levels list, etc). An appropriate model was developed on the base of the 'GKU' code. The atomic data [10] (rate coefficients, radiative probabilities, etc) were calculated by the code 'ATOM' [12] using the K-matrix (KM) method.

The main purpose of this paper is the consideration of possibilities for a construction of an $\mathrm{O}_{\text {II }}$ flux diagnostic based on visible spectral lines. Intensities of the $\mathrm{O}_{\text {II }}$ lines are defined by all collisional and radiative processes in the plasma. A collisional radiative model which is necessary for the calculation of the constants needed for the evaluation of the oxygen fluxes has been developed. To check that it is adequate an investigation of different properties of particular spectra was made and a comparison with the model was performed. The dependence of the main spectral parameters on the electron density and temperature, the type of the injected gas, etc was tested. The critical theoretical values for the flux evaluation are so-called 'ionization per photon' (IPP). The comparison of their absolute magnitudes with experimental values is particularly important. 


\section{Experiment}

TEXTOR is a medium-size tokamak (minor radius $r=0.55 \mathrm{~cm}$, major radius $R=1.75 \mathrm{~m}$ ). It has a circular poloidal cross-section and toroidal (ALT-II) limiters which determine the LCFS. In addition, there are two poloidal limiters (one upper and one lower). Each of them consists of five parts. The central block has 10 holes, through which it is possible to inject different gases [2].

$\mathrm{O}_{2}, \mathrm{CO}$ and $\mathrm{CO}_{2}$ were injected through the central block of the upper poloidal limiter (figure 1) towards the plasma centre. The poloidal limiter was positioned in the scrape-offlayer (SOL), but very close (in $1 \mathrm{~cm}$ ) to the LCFS. Before injection the gas was stored in a special reservoir $\left(\operatorname{vol} 100 \mathrm{~cm}^{3}\right.$ ). The shutter was remotely controlled. It was opened for a time of $0.3 \mathrm{~s}$ during the flat top phase of the discharge.

The spectra of $\mathrm{O}_{\text {II }}$ were registered every $20 \mathrm{~ms}$ with a spectral resolution of about $2 \AA$. The observation system had a spatial resolution of about $2 \mathrm{~mm}$ along the injection path. The emission of the injected oxygen was also registered by a 2D-camera (with the same time resolution) for the purpose of cross-calibration using the emission from the $4416 \AA$ O II line.

This cross-callibration was necessary because the spectrometer can observe simultaneously a range of about $200 \AA$ in the first order of its grating. To measure all

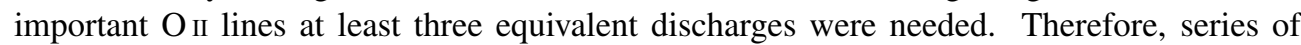
three reproducible TEXTOR discharges were performed for the different injected gases and plasma conditions.

For the evaluation of electron density and temperature, a He-beam diagnostic was used. Some series of discharges had also neutral beam injection (NBI) so that the density and the temperature of the plasma could be changed. Table 1 contains the information about the experimental series.

The pressure in the gas reservoir was measured with high time resolution. This can be used to calculate the number of injected particles from the pressure drop during the injection. The velocity of the particles was not measured directly but can be supposed to be thermal.

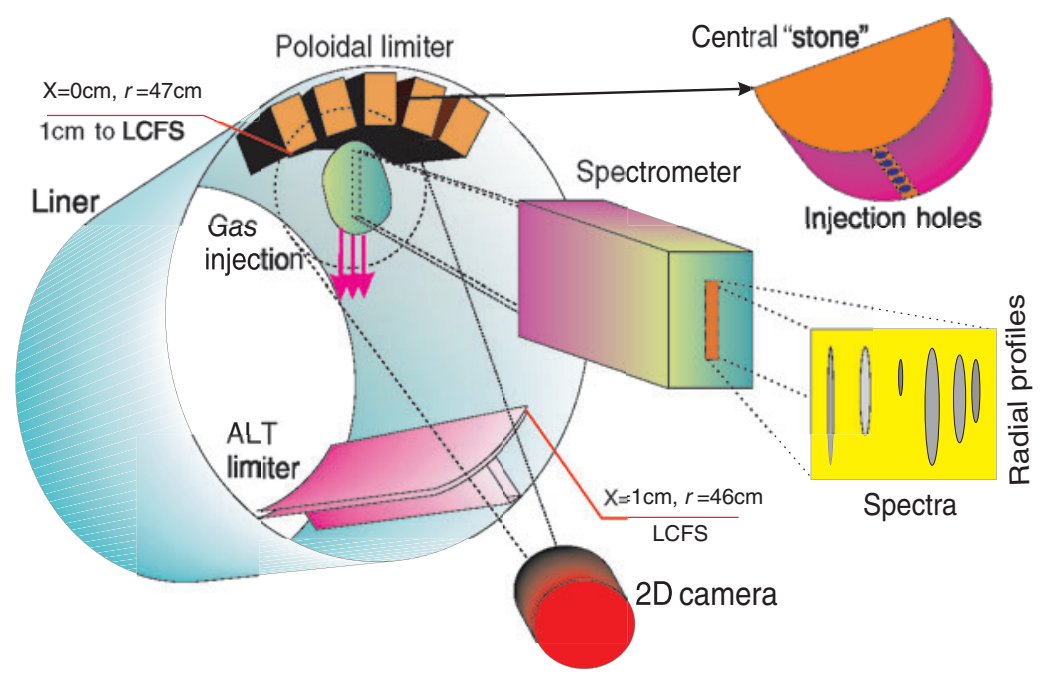

Figure 1. Scheme of the experiment. Only the upper poloidal limiter is shown. Usually for a position of different TEXTOR elements the poloidal radius $r$ is used. In our case with gas injection it is more convenient to use the $X$ coordinate along the gas path having 0 at the limiter position. 
Table 1. Experimental series.

\begin{tabular}{lllll}
\hline Serial & Shots & Gas & NBI(s), injection time & He beam diagnostic \\
\hline Ser. 1 & $89831 ; 89829 ; 89823$ & $\mathrm{O}_{2}$ & - & + \\
Ser. 2 & $90584 ; 90585 ; 90590$ & $\mathrm{CO}$ & - & + \\
Ser. 3 & $90616 ; 90617 ; 90618$ & $\mathrm{CO}$ & $2 \mathrm{MW}, 1 \mathrm{~s}$ & - \\
Ser. 4 & $91189 ; 91190 ; 91191$ & $\mathrm{CO}_{2}$ & - & + \\
Ser. 5 & $91211 ; 91213 ; 91215$ & $\mathrm{CO}_{2}$ & $2 \mathrm{MW}+1.5 \mathrm{MW}, 2 \mathrm{~s}$ & + \\
Ser. 6 & $91225 ; 91226 ; 91227$ & $\mathrm{CO}_{2}$ & $2 \mathrm{MW}+1.5 \mathrm{MW}, 2 \mathrm{~s}$ & + \\
\hline
\end{tabular}

\section{Collisional-radiative model}

The level and ion populations were calculated with the kinetic code GKU (for a short outline see [1]). The code uses an atomic data base developed for every CRM at hand (the data for every ion including the ionization rates providing connection between them are calculated by ATOM, taken from the literature or estimated by the assymptotic formulas contained in GKU). In particular, an atomic data base for the $\mathrm{O}^{+}$ion was developed.

Oxygen atoms are predominantly produced by dissociation of molecules in a thin layer near the wall. In our model this stage is not considered. We assume that the initial state is a beam of the oxygen atoms in the ground state $2 \mathrm{~s}^{2} 2 \mathrm{p}^{4} \mathrm{P}$. The last assumption was used only for modelling of the radial profiles (see section 4.3). For other calculations it was possible to use a special free parameter which was determined from the relative multiplet intensities (see section 7). The $\mathrm{O}^{+}$ions are produced by ionization of oxygen atoms and are excited by electrons afterwards. The possible approaches are: quasi-stationary (QS) and non-stationary (NS). The first one is based on the solution of the stationary balance equations set for fixed values of the electron temperature $T_{\mathrm{e}}$ and density $N_{\mathrm{e}}$. The second (NS) approach is based on the solution of the NS balance equations along the particle trajectory $x(t)$ with given profiles $T_{\mathrm{e}}(x), N_{\mathrm{e}}(x)$.

The number of photons $\lambda$ (transition $k \rightarrow k^{\prime}$ ) per ion is defined by the integral over the particle trajectory

$$
q_{\lambda}=\int N_{k}(t) A_{k k^{\prime}} \mathrm{d} t
$$

where $A_{k k^{\prime}}$ is the transition probability, $N_{k}$ is the population of the (upper) level, normalized to

$$
\sum N_{k}=1
$$

In the QS case (1) can be transformed to

$$
q_{\lambda}=\frac{W_{\lambda}}{W_{\mathrm{i}}}
$$

where $W_{\lambda}$ is the line excitation rate, obtained from solution of the stationary balance equations and $W_{\mathrm{i}}=N_{\mathrm{e}}\left\langle v \sigma_{\mathrm{i}}\right\rangle$ is the ionization rate of the ion being in consideration (more detailed see in the appendix A). The $q\left(T_{\mathrm{e}}, N_{\mathrm{e}}\right)$ should be averaged along the particle trajectory. $W_{\lambda}$ and $W_{\mathrm{i}}$ are approximately proportional to $N_{\mathrm{e}}$ and depend rather strongly on $T_{\mathrm{e}}$. However, their ratio for the cases considered in this paper is independent of $N_{\mathrm{e}}$ and only weakly dependent on $T_{\mathrm{e}}$. Therefore we can use the QS approach. The conventional IPP factor, which allows us to convert photon to particle fluxes, is defined as $1 / q$. The factor $q$ is more convenient since it is proportional to the intensity and therefore additive (over spectral lines). Small values of $q$ correspond to weak (insignificant) lines. Nevertheless, below we give values for more customary IPP factors. 


\subsection{List of levels}

The most intense $\mathrm{O}$ II lines in the visible region correspond to $3 \mathrm{p} \rightarrow 3$ s transitions. $3 \mathrm{~d} \rightarrow 3 \mathrm{p}$ lines have small branching ratios (due to transition to the ground state) or correspond to small excitation cross-sections $(\Delta L \neq \Delta l)$.

In our model SL-coupling was assumed (see appendix B). Calculations in the intermediate coupling scheme gave results reasonably close to SL-coupling. The states

$2 \mathrm{p}^{3} \mathrm{SL}, \quad 2 \mathrm{p}^{2}\left[{ }^{3} \mathrm{P}\right] n l \mathrm{SL}(n \leqslant 4), \quad 2 \mathrm{p}^{2}\left[{ }^{1} \mathrm{D},{ }^{1} \mathrm{~S}\right] n l \mathrm{SL} \quad(n \leqslant 3) \quad$ (all SL)

were included. Fine structure was not considered in most calculations. This means that fine structure levels are assumed to be populated according to their statistical weights $2 J+1$ (see section 7).

Figure 2 displays the Grotrian diagram of O II. It shows the strongest transitions including the lines observed in the experiment. The following designations are used here and below:

$$
\begin{array}{lll}
\mathrm{a}=2 \mathrm{~s}^{2} 2 \mathrm{p}^{2}\left[{ }^{3} \mathrm{P}\right] 3 \mathrm{~s} & \mathrm{~b}=2 \mathrm{~s}^{2} 2 \mathrm{p}^{2}\left[{ }^{3} \mathrm{P}\right] 3 \mathrm{p} & \mathrm{c}=2 \mathrm{~s}^{2} 2 \mathrm{p}^{2}\left[{ }^{3} \mathrm{P}\right] 3 \mathrm{~d} \\
\mathrm{a}^{\prime}=2 \mathrm{~s}^{2} 2 \mathrm{p}^{2}\left[{ }^{1} \mathrm{D}\right] 3 \mathrm{~s} & \mathrm{~b}^{\prime}=2 \mathrm{~s}^{2} 2 \mathrm{p}^{2}\left[{ }^{1} \mathrm{D}\right] 3 \mathrm{p} & \mathrm{c}^{\prime}=2 \mathrm{~s}^{2} 2 \mathrm{p}^{2}\left[{ }^{1} \mathrm{D}\right] 3 \mathrm{~d} \\
\mathrm{a}^{\prime \prime}=2 \mathrm{~s}^{2} 2 \mathrm{p}^{2}\left[{ }^{1} \mathrm{~S}\right] 3 \mathrm{~s} & \mathrm{~b}^{\prime \prime}=2 \mathrm{~s}^{2} 2 \mathrm{p}^{2}\left[{ }^{1} \mathrm{~S}\right] 3 \mathrm{p} & \mathrm{c}^{\prime \prime}=2 \mathrm{~s}^{2} 2 \mathrm{p}^{2}\left[{ }^{1} \mathrm{~S}\right] 3 \mathrm{~d} \\
4 \mathrm{a}=2 \mathrm{~s}^{2} 2 \mathrm{p}^{2}\left[{ }^{3} \mathrm{P}\right] 4 \mathrm{~s} & 4 \mathrm{~b}=2 \mathrm{~s}^{2} 2 \mathrm{p}^{2}\left[{ }^{3} \mathrm{P}\right] 4 \mathrm{p} & \left.4 \mathrm{c}=2 \mathrm{~s}^{2} 2 \mathrm{p}^{2[3} \mathrm{P}\right] 4 \mathrm{~d} \\
\ldots & \ldots & \ldots
\end{array}
$$

For example, the transition

$$
2 \mathrm{~s}^{2} 2 \mathrm{p}^{2}\left[{ }^{3} \mathrm{P}\right] 3 \mathrm{~s}\left[{ }^{4} \mathrm{P}_{3 / 2}\right]-2 \mathrm{~s}^{2} 2 \mathrm{p}^{2}\left[{ }^{3} \mathrm{P}\right] 3 \mathrm{p}\left[{ }^{4} \mathrm{~S}_{3 / 2}\right]
$$

can be written as

$$
\text { a4P4-b4S4 }
$$

These designations are convenient for the description of transitions between the states with the same atomic core, which are usually the strongest.

\subsection{Atomic data}

Atomic characteristics for some transitions in $\mathrm{O}$ II were calculated in $[6,13,14]$. In this work atomic characteristics calculated by the code ATOM [11] were used. In this code the CoulombBorn (CB) and the KM methods are used. Both are based on one-electron semi-empirical atomic wave functions with SL or intermediate couplings. The collisional rate coefficients $\langle v \sigma\rangle$ were described by fitting formulas with three adjusted parameters.

Cross-sections and rate coefficients were obtained by the KM method: 211 channels $2 \mathrm{p}^{3} \mathrm{SL}-\left[{ }^{3} \mathrm{P},{ }^{1} \mathrm{D},{ }^{1} \mathrm{~S}\right] 3 \mathrm{~s}, \mathrm{p}, \mathrm{dS} \mathrm{S}^{\prime} \mathrm{L}^{\prime}$, all possible SL, $\mathrm{S}^{\prime} \mathrm{L}^{\prime}$ were considered. The accuracy of the theoretical estimations is rather uncertain (even for strong transitions it is usually claimed to be about 10-20\%) and experimental data as well as sophisticated (CCC, R-matrix) calculations are absent. Therefore, the experimental data from TEXTOR for intensities of these lines are useful. It should be noted that for the most important excitation $2 p^{3}-2 p^{2} 3 p$ the cross-section is very large because of the monopole interaction.

3.2.1. Comparison with ADAS data. Calculated rate coefficients were compared with the data from ADAS [13]. Results are illustrated in figure 3. For transitions with $\Delta S=0$ there is a reasonable agreement. For intercombination transitions $(\Delta S=1)$ the ADAS data are much larger than those of ATOM. Calculations of the exchange amplitude are more sensitive to the details of the method. The reason for the rather large rate coefficients of the intercombination 


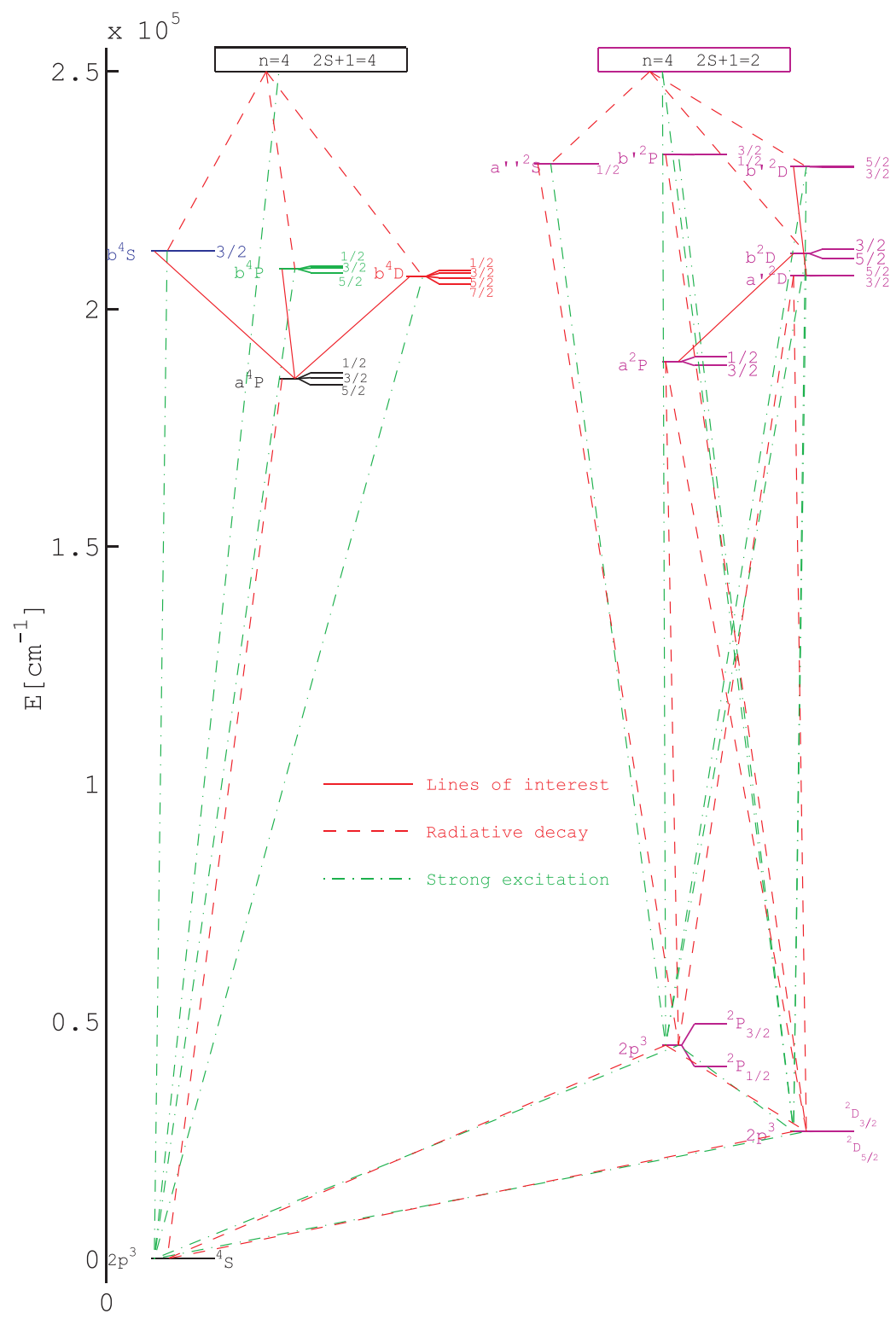

Figure 2. Grotrian diagram of the most important $\mathrm{O}$ II states.

transitions in ADAS is not clear. However, transitions of this kind play a minor role in the overall kinetic situation.

In calculations by ATOM effects of exchange (by the orthogonalized function method [12]) and channel interactions were included. On the bases of some general considerations and comparison with the TEXTOR data (see sections 7 and 4.4) we assume that the ATOM data are preferable.

The ionization cross-section from the ground term ${ }^{4} \mathrm{~S}$ was calculated with a contribution from the ionization from $2 \mathrm{p}, 2 \mathrm{~s}$ shells and excitations of autoionizing states $2 \mathrm{~s}-n l$. The calculated ionization cross-section is in good agreement with the recommended one [5], 

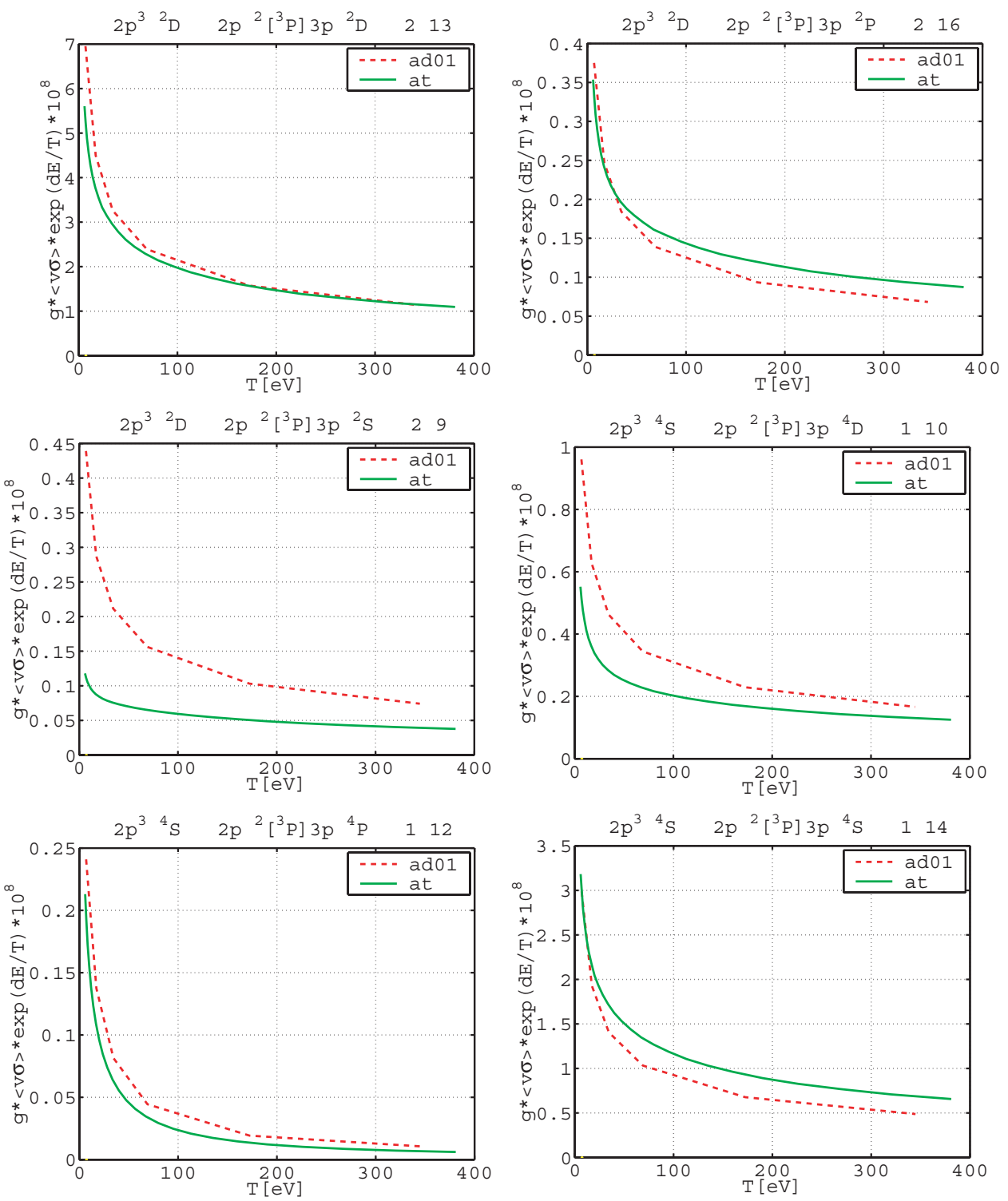

Figure 3. Rate coefficient $\left(10^{-8} \mathrm{~cm}^{3} \mathrm{~s}^{-1}\right)$. at is ATOM data; ad01 is ADAS [13] data of 2001 .

experimental data [16], and with the latest ADAS data (figure 4). Ionization from terms ${ }^{2} \mathrm{D},{ }^{2} \mathrm{P}$ was also calculated and in most cases the ionization rate averaged over terms of the ground configuration was used in the kinetic code.

\section{Spectra investigation}

\subsection{Identification of the spectral lines}

Three spectral regions were observed with 'spectral window' widths of about $200 \AA$. The emission spectra are very rich in lines from different atoms and ions. For identification purposes 

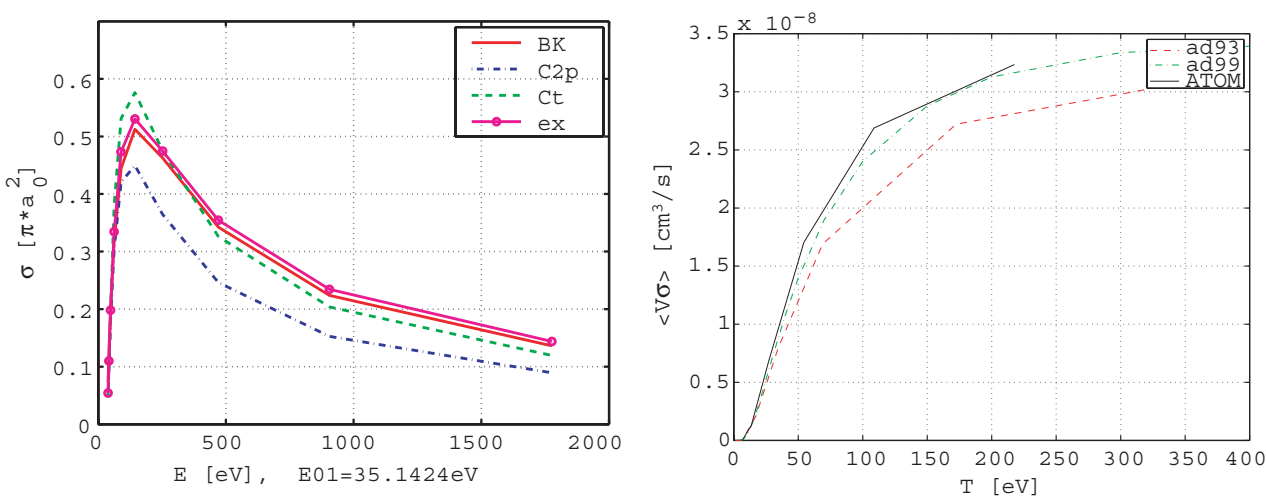

Figure 4. Ionization cross-section and rate coefficient of the $\mathrm{O}$ II $2 \mathrm{p}^{3}{ }^{4} \mathrm{~S}$ state. BK are data from [5]; ATOM data: $\mathrm{C} 2 \mathrm{p}$ is the ionization from $2 \mathrm{p}$ shell only, $C t$ is the total ionization including $2 \mathrm{~s}^{2}, 2 \mathrm{p}^{3}$ shells and autoionization; $e x$ are the experimental data [16]; $a d 93$ and $a d 99$ are ADAS [13] data of 1993 and 1999, respectively.

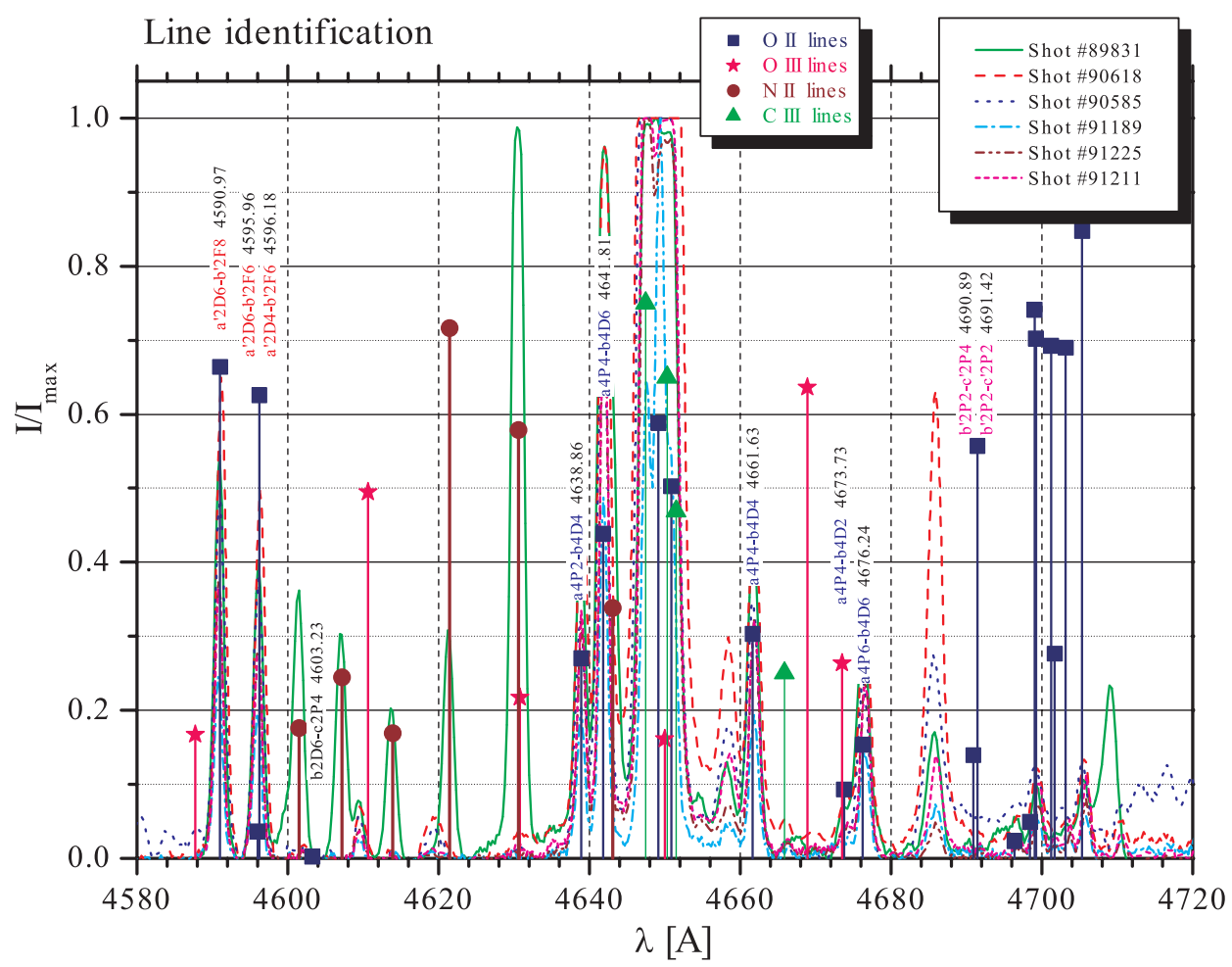

Figure 5. Spectral region ' $R$ ' $4660 \pm 100 \AA$ during injection of oxygen containing molecules.

lines with particular properties (e.g. lines of $\mathrm{N}$ II, which once appeared because of an accidential air leakage in the gas reservoir) were useful.

The region 'red' (' $R$ ', $4660 \pm 100 \AA$ ) is depicted in figure 5. Vertical lines show the position of spectral lines $k \rightarrow k^{\prime}$ with heights proportional to the $A_{k k^{\prime}}$. Wavelengths and $A_{k k^{\prime}}$ are taken from the NIST database [15]. In this region we can see components of the a4P-b4D multiplet 


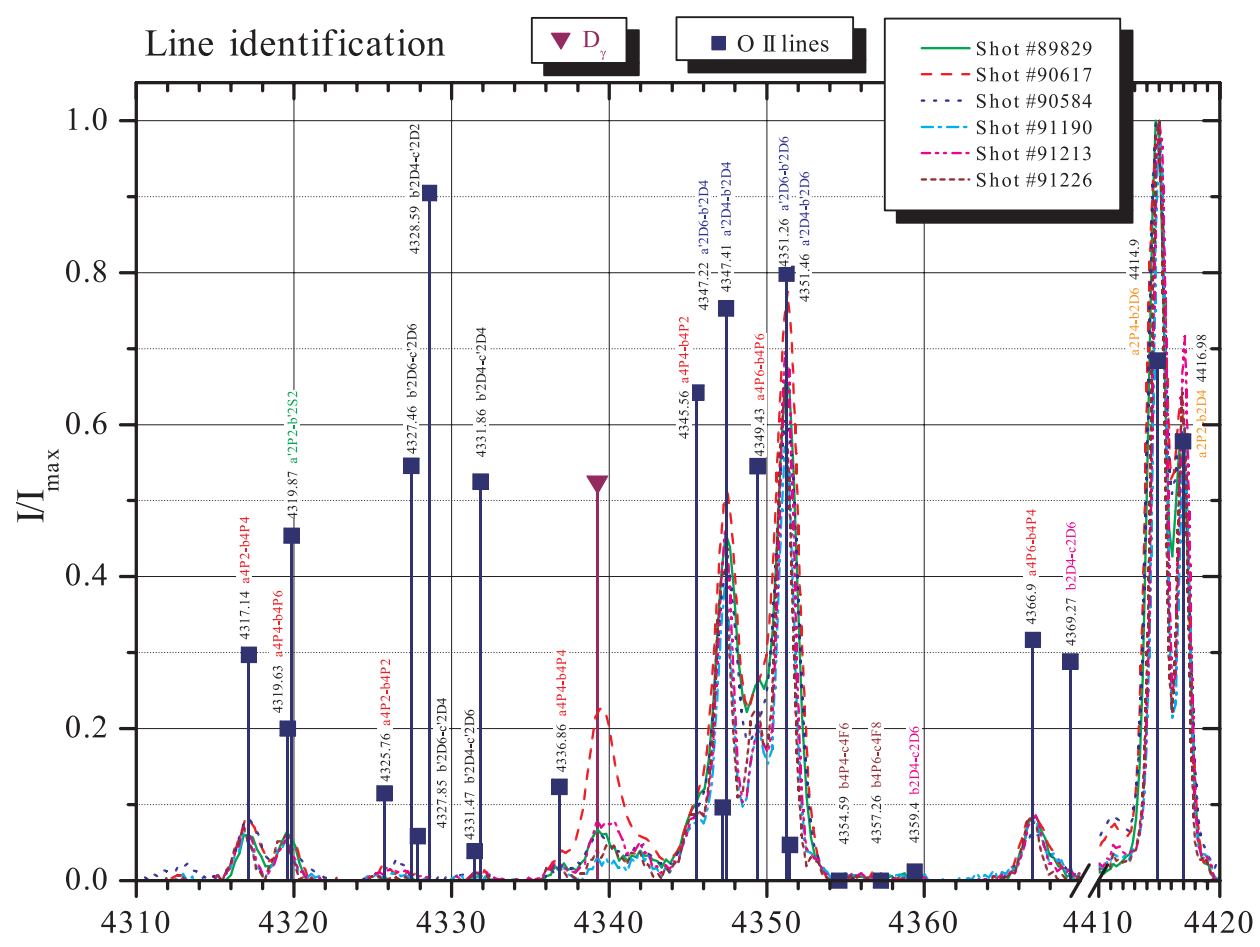

Figure 6. Spectral region 'C' $4340 \pm 100 \AA$ during injection of oxygen containing molecules.

$\left(2 \mathrm{p}^{2}\left[{ }^{3} \mathrm{P}\right] 3 \mathrm{~s}^{4} \mathrm{P}-2 \mathrm{p}^{2}\left[{ }^{3} \mathrm{P}\right] 3 \mathrm{p}^{4} \mathrm{D}\right)$. Some of them are blended with carbon lines. During discharge \#89831 strong $\mathrm{N}$ II lines were detected, which helped to confirm the line identification.

In the 'central' region ('C', $4340 \pm 100 \AA$ ) (figure 6) we can see components of the multiplets a4P-b4P $\left(2 \mathrm{p}^{2}\left[{ }^{3} \mathrm{P}\right] 3 \mathrm{~s}^{4} \mathrm{P}-2 \mathrm{p}^{2}\left[{ }^{3} \mathrm{P}\right] 3 \mathrm{p}^{4} \mathrm{P}\right)$, a2P-b2D $\left(2 \mathrm{p}^{2}\left[{ }^{3} \mathrm{P}\right] 3 \mathrm{~s}^{2} \mathrm{P}-2 \mathrm{p}^{2}\left[{ }^{3} \mathrm{P}\right] 3 \mathrm{p}{ }^{2} \mathrm{D}\right)$, $\mathrm{a}^{\prime} 2 \mathrm{D}-\mathrm{b}^{\prime} 2 \mathrm{D}\left(2 \mathrm{p}^{2}\left[{ }^{1} \mathrm{D}\right] 3 \mathrm{~s}^{2} \mathrm{D}-2 \mathrm{p}^{2}\left[{ }^{1} \mathrm{D}\right] 3 \mathrm{p}^{2} \mathrm{D}\right)$. The $D_{\gamma}$ line is observed at the centre of region. Some of lines overlap with each other. The identification was confirmed by measurements in the second order with higher spectral resolution (figure 7).

The 'blue' region ('B', $3730 \pm 100 \AA$ ) (figure 8) contains the lines of the a4P-b4S multiplet $\left(2 \mathrm{p}^{2}\left[{ }^{3} \mathrm{P}\right] 3 \mathrm{~s}^{4} \mathrm{P}-2 \mathrm{p}^{2}\left[{ }^{3} \mathrm{P}\right] 3 \mathrm{p}^{4} \mathrm{~S}\right)$ and some other $\mathrm{O}$ II and $\mathrm{O}$ III lines. Unfortunately during discharge \#91215 the strongest lines were overexposed because of the limited dynamic range of the system.

A list of the lines considered is presented in table 2.

\subsection{Dependence on $N_{\mathrm{e}}, T_{\mathrm{e}}$ and relative intensities of the multiplets}

In the QS-approach the absolute intensities are functions of $N_{\mathrm{e}}$ and $T_{\mathrm{e}}$. Figure 9 shows radial $N_{\mathrm{e}}(r)$ and $T_{\mathrm{e}}(r)$ profiles obtained by the He-beam diagnostics [9] for different series of reproducible discharges (for each series one shot was used as an example). A comparison with the radial line profiles of $\mathrm{O}$ II (see the next section) shows that the regions of interest are defined by $N_{\mathrm{e}}(r)=(1-15) \times 10^{18} \mathrm{~m}^{-3}$ and $T_{\mathrm{e}}(r)=30-200 \mathrm{eV}$. Calculations with the GKU code show only dependences on $N_{\mathrm{e}}$ and $T_{\mathrm{e}}$ for relative multiplet intensities. Examples are shown in figure 10). The very weak dependence on $N_{\mathrm{e}}, T_{\mathrm{e}}$ permits us to calculate (if necessary) the sum of intensities over a set of (overlapped) lines using simple theoretical relations. 


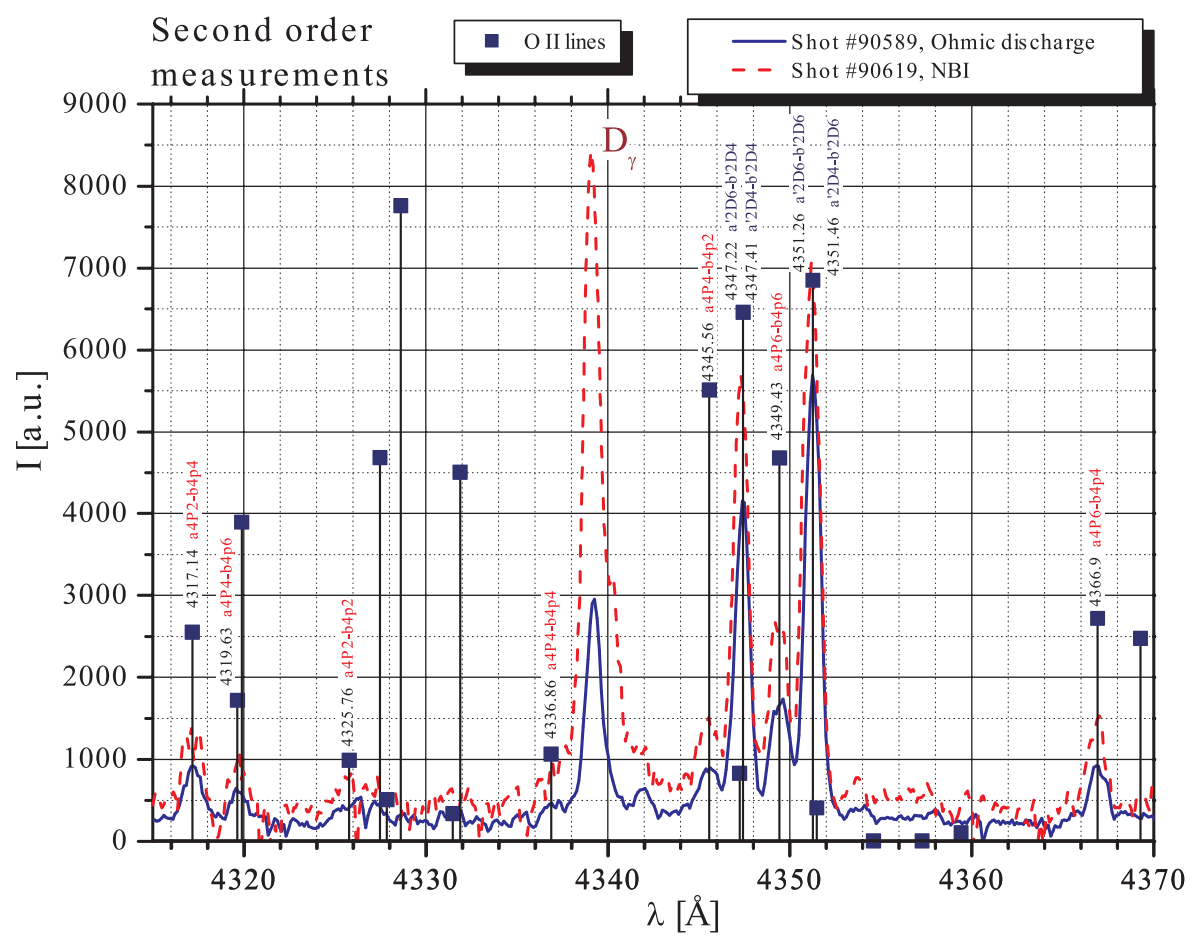

Figure 7. Spectral region 'C' $4340 \pm 100 \AA ̊$ during injection of oxygen containing molecules. Second order of the spectrometer grating.

The doublet levels are populated mainly from the doublet terms of the ground configuration and, therefore, depend on the initial population of these terms. In the QS-approach an additional assumption is required. The effect of different assumptions about the relative populations $N(\mathrm{SL})$ of the ${ }^{4} \mathrm{~S}$ and ${ }^{2} \mathrm{D},{ }^{2} \mathrm{P}$ terms was investigated. To describe them we used a fitting parameter $\alpha$. It controls additional fictitious transition rates between the states of the ground configuration which effectively represent the consequences of dissociation, ionization and propagation of the particles in the plasma before they come to a point at hand and has the following limiting values:

- $\alpha=0$-local thermodynamic equilibrium (LTR), for large $T_{\mathrm{e}} N\left({ }^{4} \mathrm{~S}\right)=0.2, N\left({ }^{2} \mathrm{D}\right)=0.5$, $N\left({ }^{2} \mathrm{P}\right)=0.3$

- $\alpha \rightarrow \infty$-the coronal limit $(\mathrm{CL}), N\left({ }^{4} \mathrm{~S}\right)=1, N\left({ }^{2} \mathrm{D}\right)=N\left({ }^{2} \mathrm{P}\right)=0$.

The experimental multiplet intensities relative to the intensity of the manifold b4S-a4P are compared with those calculated for $T_{\mathrm{e}}=90 \mathrm{eV}, N_{\mathrm{e}}=8 \times 10^{12} \mathrm{~cm}^{-3}$ and $\alpha=1-50$ in figure 11 and table 3 . Points, connected by lines, show the experimental results; separate ones show the data calculated by the GKU code.

The model predicts that the multiplet a4P-b4P is weak. Without exchange, relativistic effects and cascade intensities are zero. Including these effects in the model gives $12 \%$ of a4P-b4S intensity; the experimental ratio is less than $15 \%$. The intensities of the quartet multiplets are not sensitive to $\alpha$. We see also that the dependence on the type of gas is weak. $\alpha$ can be estimated from the doublet/quartet ratio. The optimal value of $\alpha$ is 4 . This means that the ${ }^{4} \mathrm{~S}$ ground state is populated more strongly than it would be according to its statistical weight. The intensities of the multiplets were modelled in the stationary case for a reasonable 


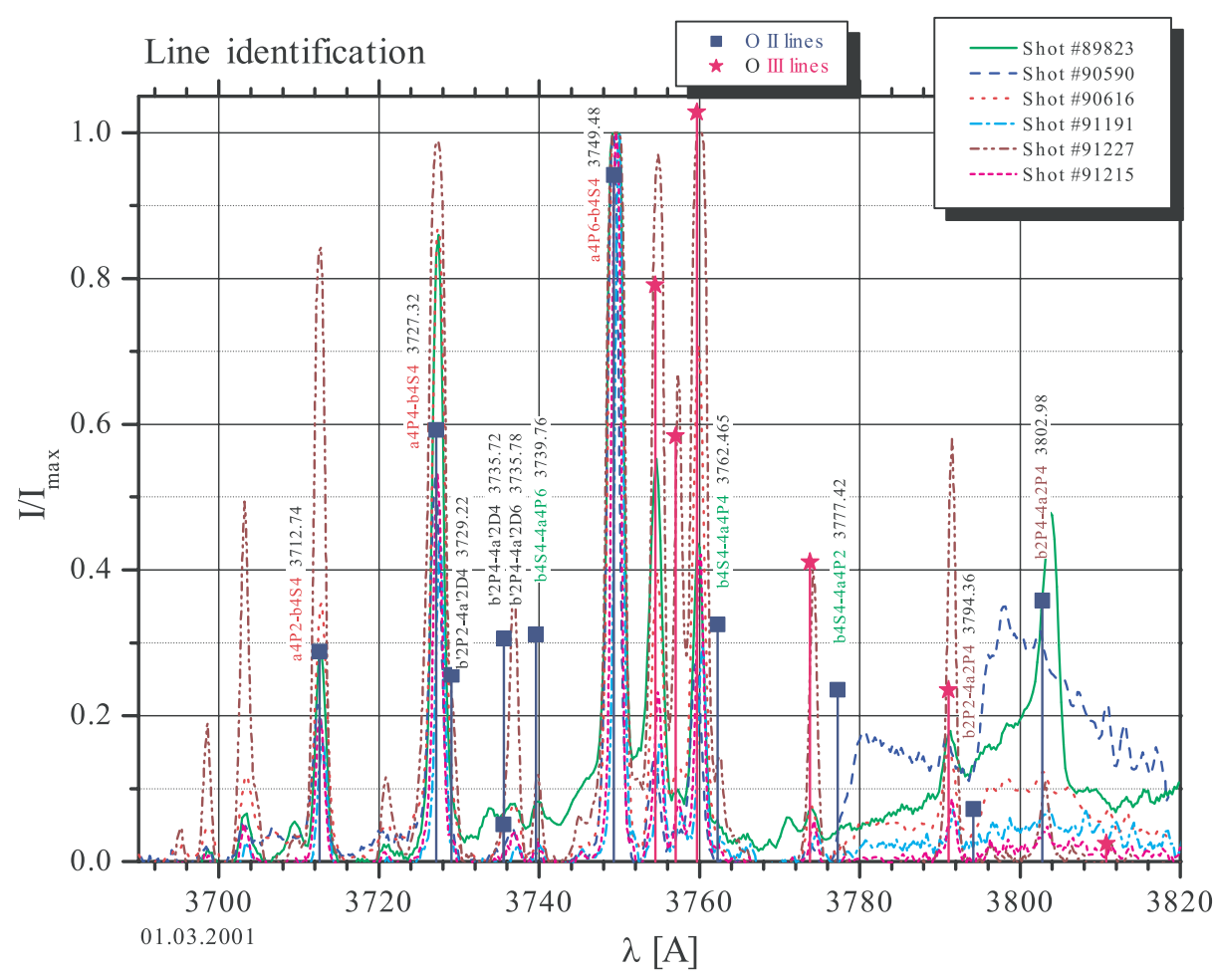

Figure 8. Spectral region 'B' $3730 \pm 100 \AA ̊$ during injection of oxygen containing molecules.

Table 2. List of considered lines.

\begin{tabular}{|c|c|c|c|c|c|c|c|}
\hline \multicolumn{6}{|c|}{$3 \mathrm{p}^{2}\left[{ }^{3} \mathrm{P}\right] 3 \mathrm{~s}-3 \mathrm{p}^{2}\left[{ }^{3} \mathrm{P}\right] 3 \mathrm{p}$} & \multirow{2}{*}{\multicolumn{2}{|c|}{$\frac{3 p^{2}\left[{ }^{1} D\right] 3 s-3 p^{2}\left[{ }^{1} D\right] 3 p}{{ }^{2} D-2} D$}} \\
\hline \multicolumn{2}{|c|}{${ }^{4} \mathrm{P}-{ }^{4} \mathrm{D}$} & \multicolumn{2}{|c|}{${ }^{4} \mathrm{P}-{ }^{4} \mathrm{P}$} & \multicolumn{2}{|c|}{${ }^{4} \mathrm{P}-{ }^{4} \mathrm{~S}$} & & \\
\hline Design. & $\lambda[\AA ̊]$ & Design. & $\lambda[\AA]$ & Design. & $\lambda[\AA]$ & Design. & $\lambda[\AA]$ \\
\hline a4P6-b4D8 & 4649.1 & a4P6-b4P6 & 4349.4 & a4P6-b4S4 & 3749.5 & $a^{\prime} 2 D 6-b^{\prime} 2 D 6$ & 4351.3 \\
\hline a4P4-b4D6 & 4641.8 & a4P4-b4P4 & 4336.9 & a4P4-b4S4 & 3727.3 & $a^{\prime} 2 D^{4}-b^{\prime} 2 D 4$ & 4347.4 \\
\hline $\mathrm{a} 4 \mathrm{P} 2-\mathrm{b} 4 \mathrm{D} 4$ & 4638.9 & $\mathrm{a} 4 \mathrm{P} 2-\mathrm{b} 4 \mathrm{P} 2$ & 4325.8 & $\mathrm{a} 4 \mathrm{P} 2-\mathrm{b} 4 \mathrm{~S} 2$ & 3712.8 & $a^{\prime} 2 D 6-b^{\prime} 2 D 4$ & 4347.2 \\
\hline a4P6-b4D6 & 4676.2 & a4P6-b4P4 & 4366.9 & \multicolumn{2}{|c|}{${ }^{2} \mathrm{P}-{ }^{2} \mathrm{D}$} & $a^{\prime} 2 D^{4}-b^{\prime} 2 D 6$ & 4351.5 \\
\hline a4P4-b4D4 & 4661.6 & $\mathrm{a} 4 \mathrm{P} 4-\mathrm{b} 4 \mathrm{P} 2$ & 4345.6 & a2P4-b2D6 & 4414.9 & & \\
\hline \multirow[t]{2}{*}{$\mathrm{a} 4 \mathrm{P} 2-\mathrm{b} 4 \mathrm{D} 2$} & 4650.8 & a4P4-b4P6 & 4319.6 & a2P2-b2D4 & 4417.0 & & \\
\hline & & a4P2-b4P4 & 4317.1 & $\mathrm{a} 2 \mathrm{P} 4-\mathrm{b} 2 \mathrm{D} 4$ & 4452.4 & & \\
\hline
\end{tabular}

density and temperature near the wall of the tokamak. The weak dependence of their ratios on these parameters was mentioned in the previous subsection.

\subsection{Radial profiles}

Radial intensity profiles along the (radial) injection direction were measured. As an example radial profiles of several lines are depicted in (figure 12). All of them were obtained during the experimental series 2 (table 1). One can see that these profiles differ only in magnitude. Their maximum positions and shapes are quite similar. 

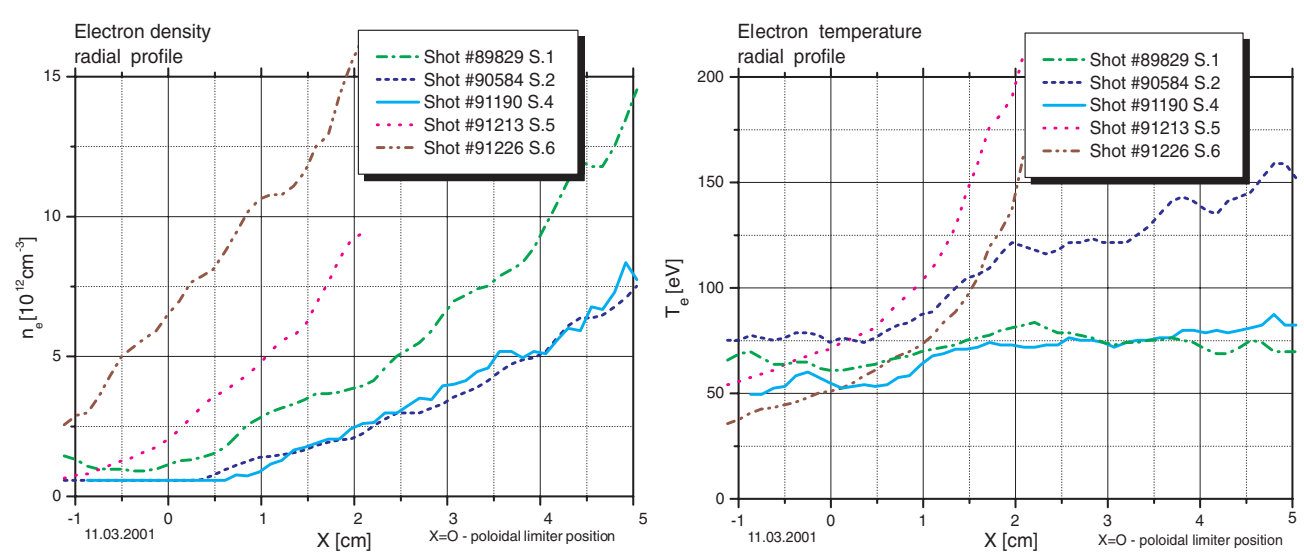

Figure 9. Electron density and temperature profiles obtained by the He beam diagnostic.
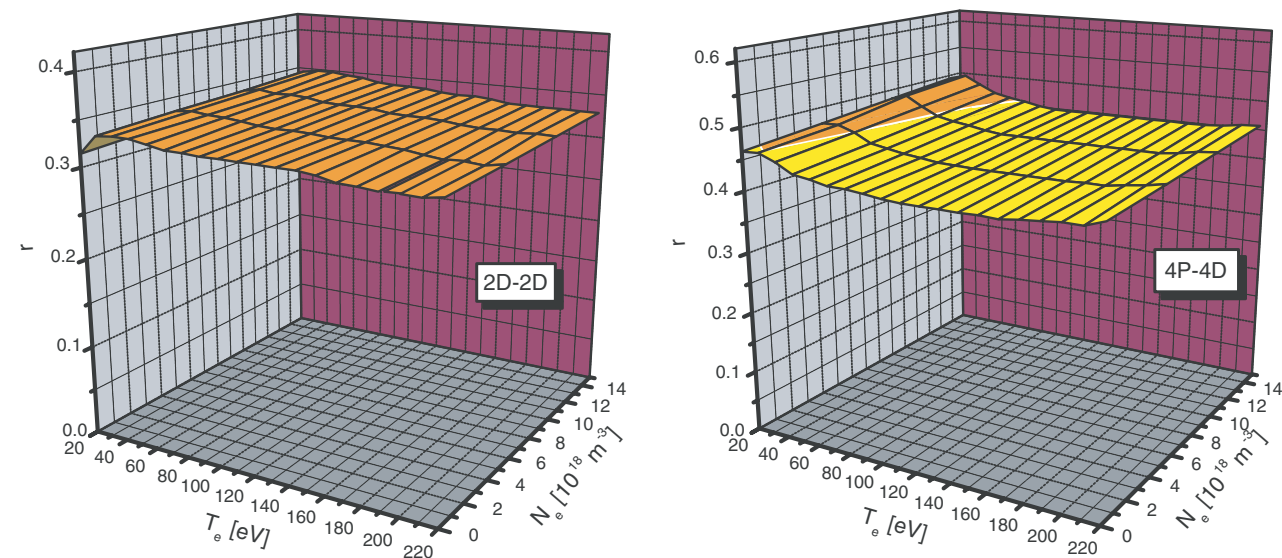

Figure 10. Intensity ratios $I(\mathrm{a} 2 \mathrm{D}-\mathrm{b} 2 \mathrm{D}) / \mathrm{I}(\mathrm{a} 4 \mathrm{~S}-\mathrm{b} 4 \mathrm{P})$ and $I(\mathrm{a} 4 \mathrm{P}-\mathrm{b} 4 \mathrm{D}) / \mathrm{I}(\mathrm{a} 4 \mathrm{~S}-\mathrm{b} 4 \mathrm{P})$ versus $T_{\mathrm{e}}$ and $N_{\mathrm{e}}$ calculated by the GKU code.

The NS approach was used to calculate the radial spectral line profiles of O II. As initial state the ground state of $\mathrm{O}_{\mathrm{I}}$ was adopted. Two different radial velocities were used for $\mathrm{O}_{\mathrm{I}}(v)$ and $\mathrm{O}_{\text {II }}\left(v_{1}\right)$ to fit the experimental profile of the $\mathrm{O}$ II lines. Satisfactory results for all considered lines are obtained with values of $v=1.1 \times 10^{3} \mathrm{~m} \mathrm{~s}^{-1}$ and $v_{1}=0.2 \times 10^{3} \mathrm{~m} \mathrm{~s}^{-1}$. It should be mentioned that $v_{1}$ must be considerably smaller than $v$, but the exact value is not important. This model corresponds to the physical situation that ions produced from neutral O practically stop their radial movement because of the strong toroidal magnetic field. $v$ is of the order of the thermal velocity corresponding to the temperature of the limiter.

In our viewing geometry (1) the observed intensity profiles are integrated along the line of sight in the toroidal direction. Therefore we can neglect the fact that ions can move quite fast along the magnetic field lines.

In figure 13 calculated and observed profiles are compared for two lines. The profiles are normalized to the maximum value. One can see that the collisional-radiative model describes the main part of the radial intensity profiles rather well. Deviations of the modelled profiles from the experimental ones are probably due to some geometrical parameters of the observation 


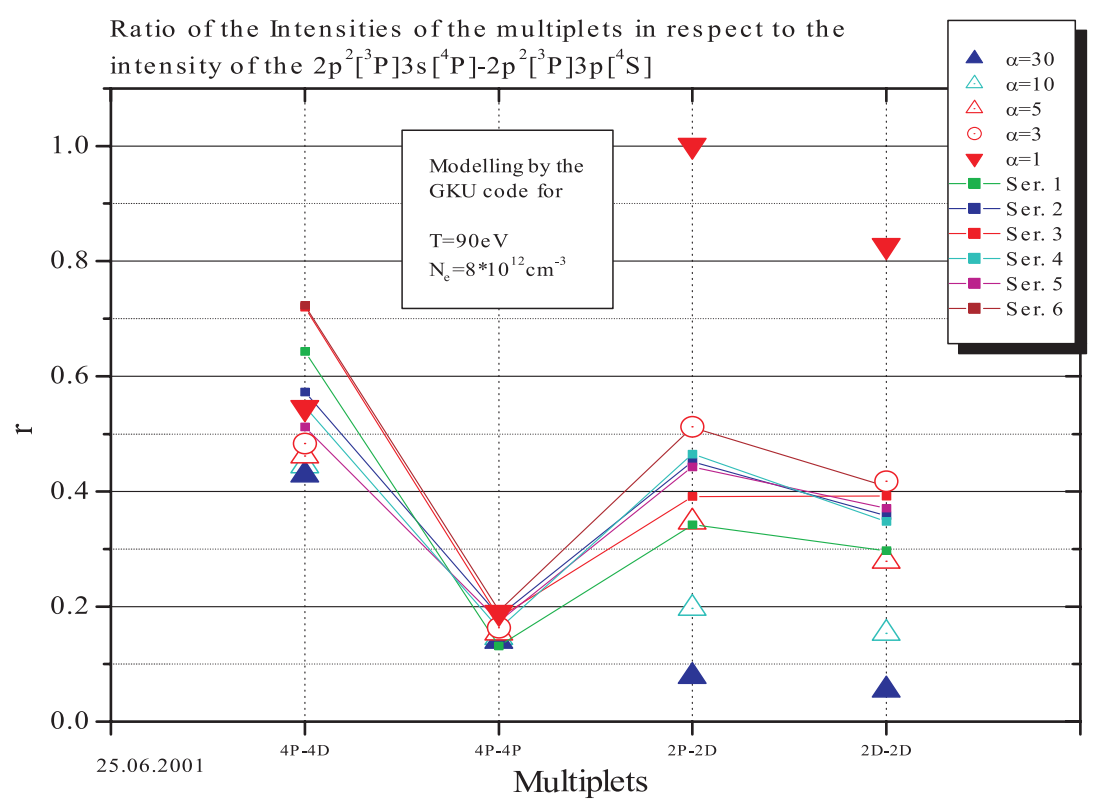

Figure 11. Ratios of the multiplet intensities.

Table 3. Multiplet intensities relative to the $2 s^{2} 2 p^{2}\left({ }^{3} \mathrm{P}\right) 3 p\left({ }^{4} S\right)-2 s^{2} 2 p^{2}\left({ }^{3} \mathrm{P}\right) 3 s\left({ }^{4} \mathrm{P}\right)$ intensity.

\begin{tabular}{|c|c|c|c|c|c|c|c|c|c|c|c|c|}
\hline \multirow[t]{2}{*}{ Trans. } & \multicolumn{6}{|c|}{ Experiment } & \multirow[b]{2}{*}{$\alpha$} & \multicolumn{5}{|c|}{ GKU } \\
\hline & Ser. 1 & Ser. 2 & Ser. 3 & Ser. 4 & Ser. 5 & Ser. 6 & & 1 & 3 & 5 & 10 & 50 \\
\hline${ }^{4} \mathrm{P}-{ }^{4} \mathrm{D}$ & 0.49 & 0.43 & 0.56 & 0.41 & 0.39 & 0.55 & & 0.52 & 0.44 & 0.42 & 0.40 & 0.37 \\
\hline${ }^{4} \mathrm{P}-{ }^{4} \mathrm{P}$ & 0.09 & 0.12 & 0.13 & 0.11 & 0.12 & 0.14 & & 0.18 & 0.15 & 0.14 & 0.13 & 0.12 \\
\hline${ }^{2} \mathrm{P}-{ }^{2} \mathrm{D}$ & 0.33 & 0.43 & 0.38 & 0.44 & 0.42 & 0.51 & & 1.00 & 0.51 & 0.34 & 0.19 & 0.05 \\
\hline${ }^{2} \mathrm{D}-{ }^{2} \mathrm{D}$ & 0.22 & 0.25 & 0.29 & 0.24 & 0.26 & 0.31 & & 0.82 & 0.41 & 0.27 & 0.15 & 0.03 \\
\hline \multicolumn{7}{|c|}{ The ground term abundance $N\left({ }^{4} \mathrm{~S}\right)$} & & 0.35 & 0.51 & 0.61 & 0.74 & 0.92 \\
\hline
\end{tabular}

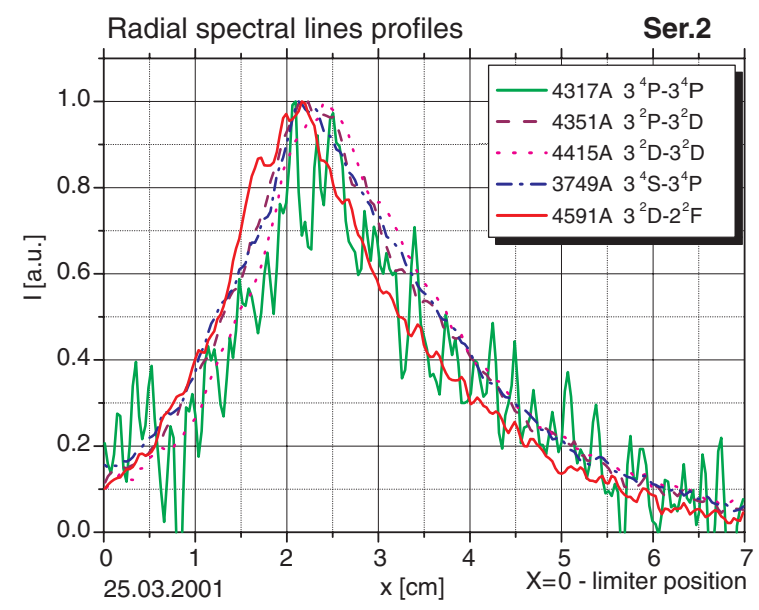

Figure 12. Radial line intensity profiles without relative intensity callibration. 

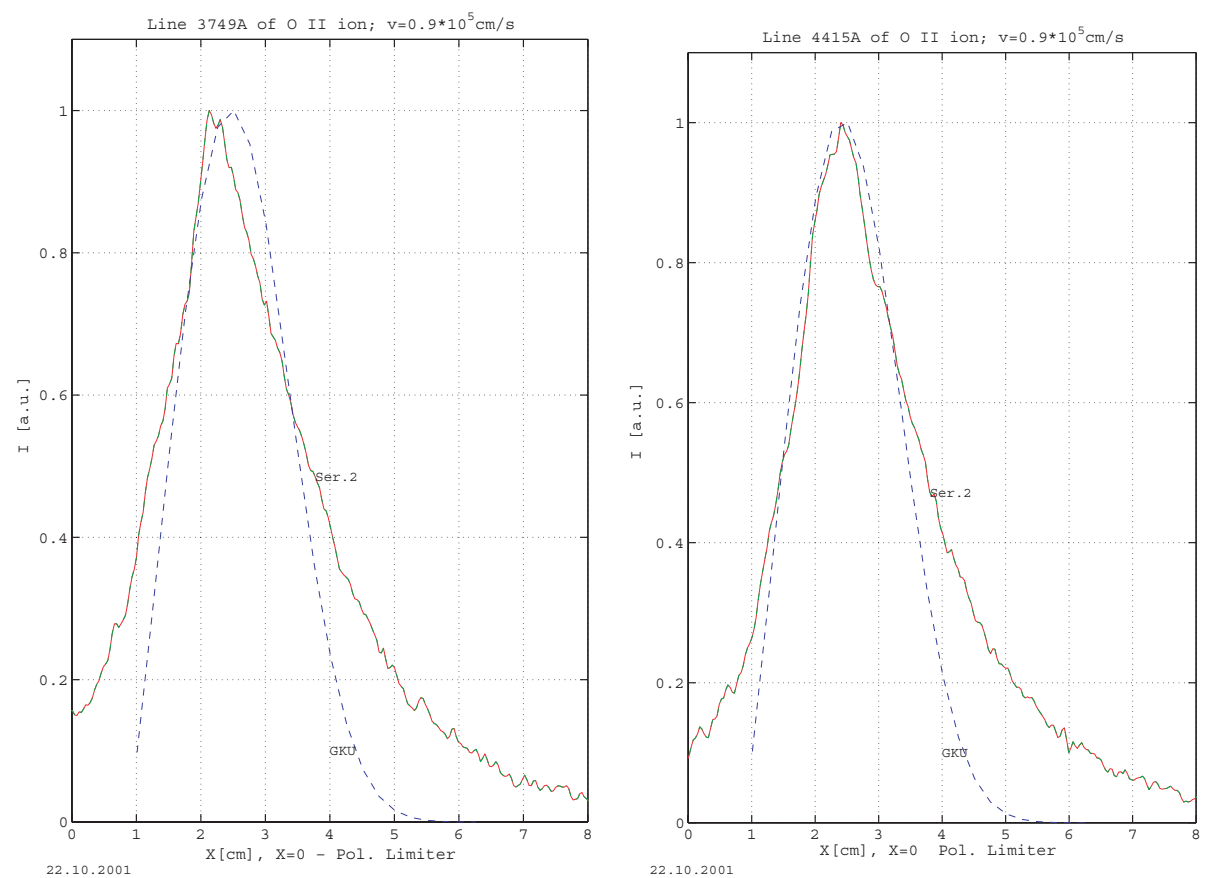

Figure 13. Line intensity radial profiles_-experimental and modelled by GKU; $X=0$ corresponds to poloidal limiter position $(R=47 \mathrm{~cm})$. LCFS was at $X=1 \mathrm{~cm}$.

system, which were not taken into account (e.g. there was a small angle between the magnetic lines and the line of sight). The velocity distribution of the particles inside the $\mathrm{O}^{+}$beam was taken to be monochromatic, which can also cause deviations. It should be mentioned that in the temperature region considered charge transfer from neutral oxygen to hydrogen $\left(\mathrm{O}+\mathrm{H}^{+} \rightarrow \mathrm{O}^{+}+\mathrm{H}\right)$ can increase the effective ionization rate by $20-30 \%$ [4]. The positions of the maximum of all calculated profiles are the same which is in agreement with the experiment.

\subsection{Absolute values}

The intensity calibration of the observation system was done using three different lamps: an Ulbricht sphere, a W-ribbon lamp and a deuterium-tungsten lamp. Over the whole region of interest the deviations between them are in the range of $20 \%$. The number of injected particles was determined via the pressure drop in the gas reservoir. After taking into account the geometrical parameters of the system one can estimate the value of IPP. The IPP values were also calculated by the GKU code on the basis of the collisional-radiative model described above. The comparison between the experimental and theoretical results is given in figure 14 and table 4. Calculated data are presented for the temperature range from 90 to $150 \mathrm{eV}$ (see figure 9), which corresponds to the temperature of the emission region for the considered lines. The dependence on the electron density is negligible.

The calculated and observed data agree in most cases within a factor 1.5. Taking into the account the different possible experimental errors (e.g. due to the recycling) we assume the accuracy of the measurements in the order of factor 2 . The accuracy of the calculated data is limited mainly by the quality of the atomic data and can be claimed to be about $20 \%$. Thus, for the estimation of the flux it is better to use the data produced by the modelling. However, it was very important to confirm the model by the comparison with the experiment. The $4 \mathrm{~S}-4 \mathrm{P}$ 


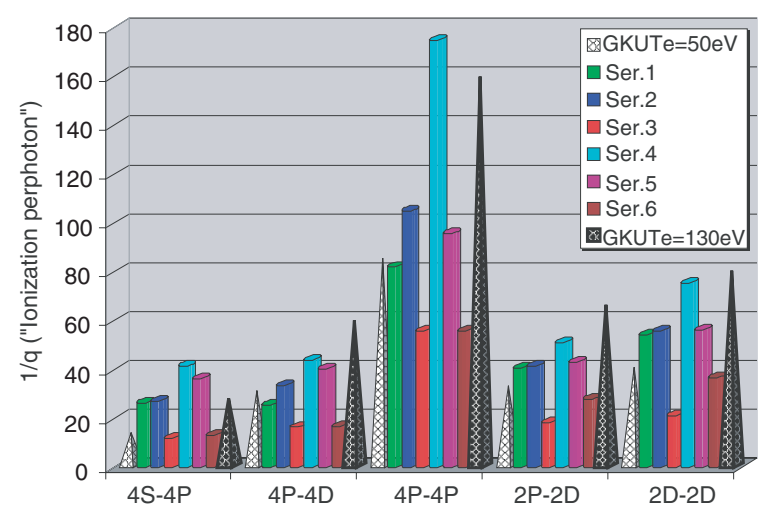

Figure 14. Comparison of the experimental and the modelled 'IPP'.

Table 4. IPP for considered multiplets.

\begin{tabular}{lllccc}
\hline Multiplet & $\mathrm{b} 4 \mathrm{~S}-\mathrm{a} 4 \mathrm{P}$ & $\mathrm{b} 4 \mathrm{D}-\mathrm{a} 4 \mathrm{P}$ & $\mathrm{b} 4 \mathrm{P}-\mathrm{a} 4 \mathrm{P}$ & $\mathrm{b} 2 \mathrm{D}-\mathrm{a} 2 \mathrm{P}$ & $\mathrm{b}^{\prime} 2 \mathrm{D}-\mathrm{a}^{\prime} 2 \mathrm{D}$ \\
\hline GKU $T_{\mathrm{e}}=50 \mathrm{eV}$ & 14 & 30 & 85 & 33 & 40 \\
Ser. 1 & 28 & 27 & 88 & 43 & 54 \\
Ser. 2 & 24 & 64 & 165 & 55 & 68 \\
Ser. 3 & 23 & 32 & 63 & 35 & 41 \\
Ser. 4 & 42 & 44 & 175 & 51 & 76 \\
Ser. 5 & 37 & 40 & 96 & 143 & 56 \\
Ser. 6 & 14 & 18 & 64 & 30 & 39 \\
GKU $T_{\mathrm{e}}=130 \mathrm{eV}$ & 27 & 59 & 159 & 66 & 80 \\
\hline
\end{tabular}

quartet lies in the UV region which is more difficult for experimental observation. The error in evaluation of the absolute values is larger than for the relative ones because of contributions from the averaging procedure, error of geometric and measured parameters (e.g. the pressure drop in the gas reservoir, which is essential for the estimation the number of particles ), etc. Also it should be mentioned, that the data processing was optimized either for absolute or for relative measurements (e.g. for the relative values if we assume the apparatus function to be constant it is better to take the spectral, radial and time zone near the maximum to decrease the errors).

The IPP value for the $\mathrm{a}^{\prime} 2 \mathrm{D}-\mathrm{b}^{\prime} 2 \mathrm{D}$ multiplet was compared with a value obtained via a cross-

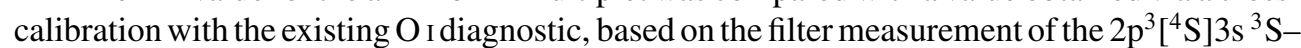
$2 p^{3}\left[{ }^{4} \mathrm{~S}\right] 3 \mathrm{p}^{3} \mathrm{P}(8450 \AA) \mathrm{O}$ I multiplet intensity (black body radiation from hot tokamak parts was found to be negligible). The IPP value of 32 obtained by this method for the $\mathrm{O}$ II $\mathrm{a}^{\prime} 2 \mathrm{D}-\mathrm{b}^{\prime} 2 \mathrm{D}$ multiplet is in an agreement with those presented in table 4 . The IPP values for the O I multiplet were taken from [7]. In this work the IPP for $\mathrm{a}^{\prime} 2 \mathrm{P}-\mathrm{b}^{\prime} 2 \mathrm{D}$ line was also presented (about 10).

One interesting observation is the possible dependence of the IPP values on the NBI power. One can see that the smallest IPP's were obtained during the series 3 and 6 when neutral beams were in operation. In the series from 4 to 6 the IPP decreases and the NBI increases. The reason for such a dependence is not yet clear.

\section{Conclusion}

Spectra of $\mathrm{O}_{\text {II }}$ in the TEXTOR boundary plasma were obtained and identified. The absolute line intensities of 5 multiplets were measured and the corresponding IPP factors are obtained. 
A collisional-radiative model for the $\mathrm{O}_{\text {II }}$ ion was developed in QS and NS approach (code GKU). The atomic database was mainly obtained by the ATOM-AKM code.

The calculated relative intensities of multiplets are in reasonable agreement with the measured ones. The calculated and measured IPP factors are in agreement within a factor of 1.5. The model has been proved to be capable of explaining other $\mathrm{O}$ II spectra features, such as relative line intensities or population of the ground configuration states. This makes it possible to recommend the calculated values for evaluations of the $\mathrm{O}$ flux in tokamaks.

It was shown that the IPP factors provided in this paper have a weak dependence on $N_{\mathrm{e}}$, particle velocities and the type of injected gas $\left(\mathrm{CO}, \mathrm{CO}_{2}\right.$, and $\mathrm{O}_{2}$ were used during the experiment). The IPP values in the range from 50 to $130 \mathrm{eV}$ change within a factor of 2 . The data presented in table 4 can be used for the evaluation of the oxygen flux (using interpolation if additional information on the electron temperature is available). The dependence on the electron density can be neglected.

The lines applicable for an interference filter diagnostic should be rather strong and isolated (a convenient filter transmission 'window' is about $30 \AA$ ). The a4P-b4S lines 3712 and $3727 \AA$ between quartet states satisfy these conditions, however, they lie in the near UV spectral region. The a2P-b2D lines 4415 and $4417 \AA$ of the doublet system also satisfy the conditions, but they have a dependence on the populations of the metastable ${ }^{2} \mathrm{D}$ and ${ }^{2} \mathrm{P}$ levels of the ground configuration. In principle it is possible to use the a4P-b4P lines 4317 and $4319 \AA$, however they are rather weak. Thus, a combination of the a4P-b4S and a2P-b2D lines seems to be the preferred choice.

\section{Acknowledgments}

This work was supported in part (D Borodin, I Beigman and L Vainschtein) by RFBR (project 00-02-17825) and PICS-RFBR-98-02-22027.

This work is the part of PhD-theses of D Borodin.

We thank Dr Th Greenland, Dr G Sergienko and Dr Ph Mertens for useful discussions and remarks.

\section{Appendix A. Derivation of the relation for the number of photons}

Let us consider a particle incoming to hot plasma at time $t=0$. We assume that the plasma temperature $T_{\mathrm{e}}$ is larger than the equilibrium temperature of the considered ions. In this case recombination processes are negligible. The relative concentration of the ions with spectroscopic symbol $Z$ along the trajectory can be described by the system of equations:

$$
\frac{\mathrm{d}}{\mathrm{d} t} N_{Z}=-N_{Z} W_{Z}+N_{Z-1} W_{Z-1}
$$

where $W_{Z} \equiv W(Z \rightarrow Z+1)$ is the ionization rate. $W_{Z} \equiv 0$ if $Z<1$ or $Z>Z_{\mathrm{n}}+1\left(Z_{\mathrm{n}}\right.$ is the nuclear charge). As the initial value we have $N_{Z}(t=0)=\delta(Z, 1)$. We obtain from this system for ions with $1<Z<Z_{n}+1$

$$
\int_{0}^{\infty} N_{Z} W_{Z} \mathrm{~d} t=\int_{0}^{\infty} N_{Z-1} W_{Z-1} \mathrm{~d} t=1 .
$$

In the frame of the QS approach the number of the photons with wavelength $\lambda$ corresponding to the $k \rightarrow k^{\prime}$ transition can be written in the form:

$$
q_{\lambda}=\int_{0}^{\infty} N_{k}(t) A\left(k \rightarrow k^{\prime}\right) \mathrm{d} t \approx \int_{0}^{\infty} N_{Z} W_{\lambda}\left(T_{\mathrm{e}}, N_{\mathrm{e}}\right) \mathrm{d} t
$$


where $W_{\lambda}\left(T_{\mathrm{e}}, N_{\mathrm{e}}\right)$ is the effective rate of the $k \rightarrow k^{\prime}$ transition. If the ratio $W_{\lambda} / W_{Z}$ does not change along the particle trajectory in the region which gives main contribution in the integral then using (5) we obtain

$$
q_{\lambda}=\int_{0}^{\infty} \frac{W_{\lambda}\left(T_{\mathrm{e}}, N_{\mathrm{e}}\right)}{W_{Z}} N_{Z} W_{Z} \mathrm{~d} t \approx \frac{W_{\lambda}\left(T_{\mathrm{e}}, N_{\mathrm{e}}\right)}{W_{Z}} .
$$

Thus, the relation $q_{\lambda}=W_{\lambda}\left(T_{\mathrm{e}}, N_{\mathrm{e}}\right) / W_{Z}$ is based on three assumptions: neglecting the recombination processes (5), QS approach (6), and almost constant $W_{\lambda} / W_{Z}$ ratio.

\section{Appendix B. Relative intensities inside multiplets}

It is reasonable to suppose that the upper multiplet levels are populated proportional to their statistical weights (as will be tested by comparison with the experimental data). Thus, the intensity of the fine structure component $\left(J \rightarrow J^{\prime}\right)$ will be proportional to $(2 J+1) A_{J J^{\prime}}$ and the relative intensity to the sum of all observed fine structure components.

$$
r\left(J \rightarrow J^{\prime}\right)=\frac{I_{J J^{\prime}}}{\sum_{J J^{\prime}} I_{J J^{\prime}}}=\frac{(2 J+1) A_{J J^{\prime}}}{\sum_{J J^{\prime}}(2 J+1) A_{J J^{\prime}}}
$$

Calculated relative intensities are compared with the experimental ones in tables 5-7 and figures $15-17$.

The calculated data correspond to SL-coupling. One can see a rather good agreement between the experimental and calculated data. This justifies the use of SL-coupling and the assumption that the $J$ levels are statistically populated.

\begin{tabular}{|c|c|c|c|c|c|c|c|}
\hline \multicolumn{2}{|c|}{ Spectral lines } & \multicolumn{6}{|c|}{$r$} \\
\hline$\lambda, \AA$ & Transition & Calc. & Ser. 1 & Ser. 2 & Ser. 3 & Ser. 4 & Ser. 5 \\
\hline 3712.7 & a4P2-b4S4 & 0.16 & 0.13 & 0.12 & 0.16 & 0.10 & 0.12 \\
\hline 3727.3 & $\mathrm{a} 4 \mathrm{P} 4-\mathrm{b} 4 \mathrm{~S} 4$ & 0.32 & 0.40 & 0.30 & 0.39 & 0.27 & 0.30 \\
\hline 3749.5 & $\mathrm{a} 4 \mathrm{P} 4-\mathrm{b} 4 \mathrm{~S} 4$ & 0.52 & 0.47 & 0.57 & 0.45 & 0.62 & 0.58 \\
\hline
\end{tabular}

Table 5. Relative intensities of $2 \mathrm{~s}^{2} 2 \mathrm{p}^{2}\left({ }^{3} \mathrm{P}\right) 3 \mathrm{p}\left({ }^{4} \mathrm{~S}\right)-2 \mathrm{~s}^{2} 2 \mathrm{p}^{2}\left({ }^{3} \mathrm{P}\right) 3 \mathrm{~s}\left({ }^{4} \mathrm{P}\right)$.

\begin{tabular}{|c|c|c|c|c|c|c|c|}
\hline \multicolumn{2}{|c|}{ Spectral lines } & \multicolumn{6}{|c|}{$r$} \\
\hline$\lambda, \AA$ & Transition & Calc. & Ser. 1 & Ser. 2 & Ser. 3 & Ser. 4 & Ser. 5 \\
\hline 4317.14 & a4P2-b4P4 & 0.325 & 0.29 & 0.43 & 0.37 & 0.32 & 0.34 \\
\hline 4319.63 & a4P4-b4P6 & 0.329 & 0.31 & 0.23 & 0.25 & 0.27 & 0.26 \\
\hline 4366.9 & a4P6-b4P4 & 0.346 & 0.40 & 0.34 & 0.38 & 0.41 & 0.40 \\
\hline
\end{tabular}

Table 6. Relative intensities of $2 s^{2} 2 p^{2}\left({ }^{3} \mathrm{P}\right) 3 \mathrm{p}\left({ }^{4} \mathrm{P}\right)-2 \mathrm{~s}^{2} 2 \mathrm{p}^{2}\left({ }^{3} \mathrm{P}\right) 3 \mathrm{~s}\left({ }^{4} \mathrm{P}\right)$.

\begin{tabular}{|c|c|c|c|c|c|c|c|}
\hline \multicolumn{2}{|c|}{ Spectral lines } & \multicolumn{6}{|c|}{$r$} \\
\hline$\lambda, \AA$ & Transition & Calc. & Ser. 1 & Ser. 2 & Ser. 3 & Ser. 4 & Ser. 5 \\
\hline 4638.86 & a4P2-b4D4 & 0.19 & 0.21 & 0.20 & 0.23 & 0.20 & 0.21 \\
\hline 4641.81 & a4P4-b4D6 & 0.45 & 0.45 & 0.47 & 0.42 & 0.48 & 0.46 \\
\hline 4661.63 & a4P4-b4D4 & 0.21 & 0.20 & 0.20 & 0.21 & 0.18 & 0.19 \\
\hline 4676.24 & a4P6-b4D6 & 0.16 & 0.14 & 0.13 & 0.14 & 0.13 & 0.13 \\
\hline
\end{tabular}

Table 7. Relative intensities of $2 \mathrm{~s}^{2} 2 \mathrm{p}^{2}\left({ }^{3} \mathrm{P}\right) 3 \mathrm{p}\left({ }^{4} \mathrm{D}\right)-2 \mathrm{~s}^{2} 2 \mathrm{p}^{2}\left({ }^{3} \mathrm{P}\right) 3 \mathrm{~s}\left({ }^{4} \mathrm{P}\right)$. 


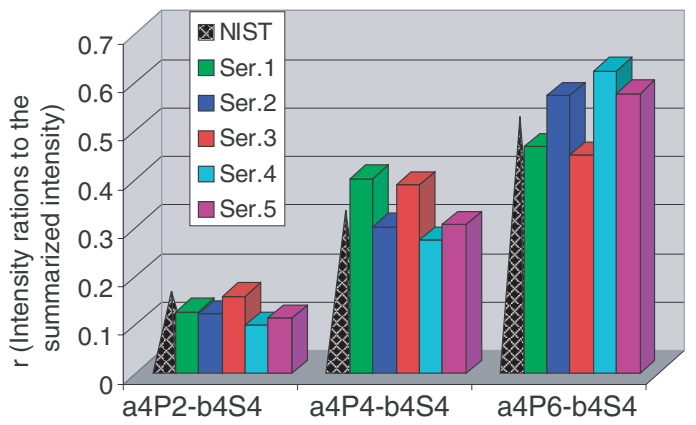

Figure 15. Relative intensities inside the ${ }^{4} \mathrm{P}-{ }^{4} \mathrm{~S}$ multiplet.

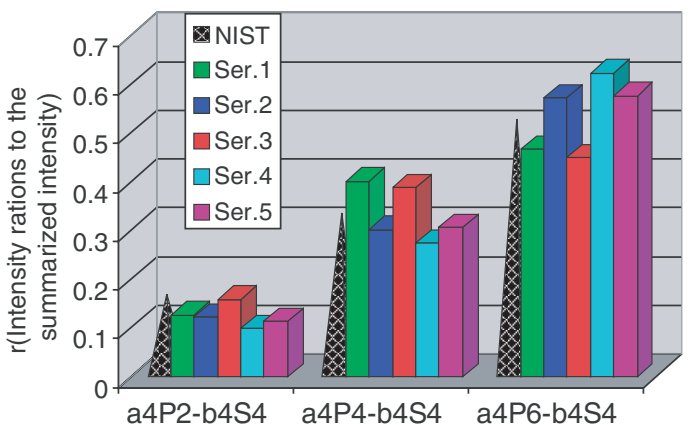

Figure 16. Relative intensities inside the ${ }^{4} \mathrm{P}-{ }^{4} \mathrm{~S}$ multiplet.

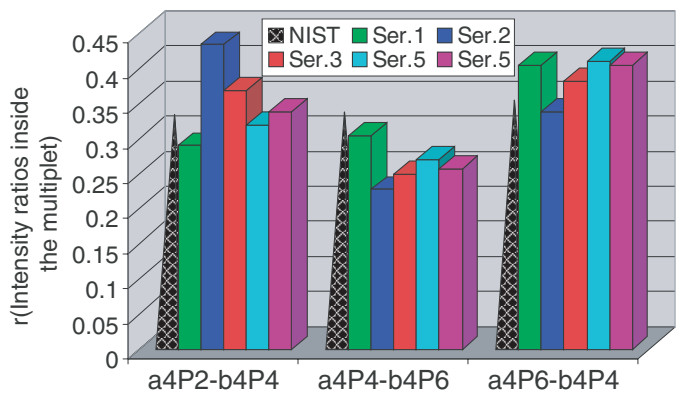

Figure 17. Relative intensities inside the ${ }^{4} \mathrm{P}-{ }^{4} \mathrm{P}$ multiplet.

\section{References}

[1] Beigman I L, Kocsis G, Pospieszczyk A and Vainshtein L A 1998 The line emission of carbon and lithium beams in the plasma edge of TEXTOR Control. Fusion and Plasma Phys. 40 1689-705

[2] Bogen P, Hartwig H, Hintz E, Hoether K, Lie Y T, Pospieszczyk A, Samm U and Bieger W 1984 Properties of the TEXTOR boundary layer J. Nucl. Mater. 128\&129 157-62

[3] Hey J D, Chu C C, Brezinsek S, Mertens Ph and Untenberg B Oxygen ion impurity in the TEXTOR tokamak boundary plasma observed and analysed by Zeeman spectroscopy J. Phys. B submitted

[4] Janev R K and Smith J J 1993 J. Nucl. Fusion Suppl. 477

[5] Lennon M A, Bell K L, Gilbody H B, Hughes J H, Kingston A E, Murray M J and Smith F J 1988 J. Phys. Chem. Ref. Data 171285

[6] McLaughlin B M and Bell K L 1993 Aph. J. 408753

Bell K L, Hibbert A, Stafford and McLaughlin B M 1994 Phys. Scr. 50343 
[7] Pospieszczyk A 1993 Diagnostic of edge plasmas by optical method Atomic and Plasma-Material Processes in Controlled Thermonuclear Fusion ed R K Janev and H W Darwin (Amsterdam: Elsevier) p 213

[8] Samm U and the TEXTOR-94 Team 1999 Progress in plasma-wall-interaction research-contributions from TEXTOR-94 Plasma Phys. Control. Fusion 41 B57-76

[9] Schweer B, Brix M and Lehnen M 1999 Measurement of edge parameters in TEXTOR-94 at the low and high field side with atomic beams J. Nucl. Matter 266-269 673

Brix M 1999 PhD Thesis Forschungszentrum Juelich, Juel-3638

[10] Shevelko V P 1997 Atoms and their spectroscopic properties, Springer Series on Atoms and Plasmas, vol 18

[11] Shevelko V P and Vainshtein L A 1993 Atomic Physics for Hot Plasmas (IOP)

[12] Sobelman I I, Vainshtein L A and Yukov E A 1995 Excitation of Atoms and Broadening of Spectral Lines, Springer Series on Atoms and Plasmas (Berlin: Springer), vol 1

[13] Summers H P and von Hellermann M 1993 Atomic and Plasma-Material Interaction Processes in Controlled Thermonuclear Fusion ed R K Janev and H W Drawin (Amsterdam: Elsevier) pp 87-117; Atomic Data Analysis Structure, see also http://adas.phys.strath.ac.uk

[14] Tayal S S and Richardson L M 2000 J. Phys. B 33443

[15] Wiese W L, Fuhr J R and Deters T M 1996 Atomic Transition Probabilities of Carbon, Nitrogen, and Oxygen. A critical Data Compilation, Monograph No 7 (Washington, DC: AIP)

[16] Yamada I et al 1988 J. Phys. Soc. Japan 572699 